\title{
Comparison of three dimensional magnetic resonance imaging in conjunction with a blood pool contrast agent and nuclear scintigraphy for the detection of experimentally induced gastrointestinal bleeding
}

\author{
P R Hilfiker, D Weishaupt, G M Kacl, F H Hetzer, M D Griff, S G Ruehm, J F Debatin
}

\begin{abstract}
Background and aims-To compare the performance of $3 \mathrm{D}$ magnetic resonance imaging (MRI) in conjunction with an intravascular contrast agent with that of scintigraphy, with respect to detection and localisation of gastrointestinal haemorrhage in vivo in pigs.

Methods-Intraluminal bleeding sites were surgically created in the small bowel and colon of six pigs. The animals underwent scintigraphy with ${ }^{99 \mathrm{~m}}$ Tc labelled red blood cells and 3D MRI following administration of an intravascular contrast agent (NC100150) at five minute intervals over 30 minutes. For analysis, the intestinal tract was divided into six segments. Based on the two evaluated methods, each segment was characterised on a five point scale regarding the presence of a bleed. At autopsy, the surgically manipulated bowel segments were inspected for the presence of haemorrhage.
\end{abstract}

Results-Bleeding was confirmed at autopsy in 18/36 segments. Contrast extravasation with subsequent movement through the bowel could be documented on MRI data sets. All segments were correctly characterised, resulting in $100 \%$ sensitivity and specificity for MRI. Based on scintigraphy, interpretation of seven segments (19\%) was false (sensitivity/ specificity of $78 \% / 72 \%$ ). Differences in diagnostic performance were evident in the receiver operator characteristic (ROC) analysis, with an area under the MRI curve of 0.99 and under the scintigraphy curve of 0.85 .

Conclusion-In conjunction with an intravascular contrast agent, $3 \mathrm{D}$ MRI permits accurate detection and localisation of gastrointestinal bleeding. The extent and evolution of intestinal bleeding can be determined with repeated data acquisition.

(Gut 1999;45:581-587)

Keywords: gastrointestinal tract; haemorrhage; scintigraphy; magnetic resonance; contrast agent

Dr J F Debatin, Institute of Diagnostic Radiology, University Hospital Zürich, Rämistr. 100, CH-8091 Zürich, Switzerland.

Accepted for publication 14 April 1999
In the vast majority of the 300000 patients admitted to hospitals in the United States for gastrointestinal haemorrhage, the bleeding site is readily identified and usually successfully treated with endoscopy. ${ }^{1}$ Despite panendoscopy, the location of the bleeding site remains unknown in $5-10 \%$ of patients, who generally present with symptoms of chronic severe anaemia and/or relapsing melaena. ${ }^{23}$ The small bowel and proximal colon are most frequently implicated in these cases. Rapid localisation of the bleeding site is of paramount importance for choosing and delivering effective therapy. Currently, a variety of diagnostic tests are used alone or in combination in patients with gastrointestinal haemorrhage of unknown origin. These include endoscopy/enteroscopy, ${ }^{4}$ angiography, ${ }^{56}$ computed tomography, ${ }^{7}$ and scintigraphy. ${ }^{89}$ Accordingly, the diagnostic work up of these patients is often time consuming, and frequently associated with considerable patient discomfort as well as high cost. Despite the use of all these methods, the site and nature of gastrointestinal bleeding remains obscure in a considerable number of patients. $^{10}$

Ideally, the technique for detecting and localising gastrointestinal bleeding should be non-invasive, highly sensitive and specific, spatially accurate, and quick to perform. To detect intermittent bleeding, sequential examinations must be possible. We recently described an approach based on the use of an intravascular paramagnetic contrast agent in combination with fast 3D magnetic resonance imaging (MRI). ${ }^{11}$ The technique is based on the following concept: any disruption of vascular integrity within the bowel results in extravasation of the intravascular agent with subsequent accumulation in the intestinal lumen. In vitro studies have shown the proposed technique to be as sensitive as nuclear scintigraphy. ${ }^{11}$

The purpose of this study was to compare the performance of 3D MRI in conjunction with an intravascular contrast agent with that of nuclear scintigraphy, with respect to detection and localisation of gastrointestinal haemorrhage under in vivo conditions in pigs. Macropathology was used as the standard of reference.

Abbreviations used in this paper: GRE, gradient recalled echo; MIP, maximum intensity projections; MRI, magnetic resonance imaging; RBC, red blood cell; ROC, receiver operator characteristic; SPECT, single photon emission computed tomography. 


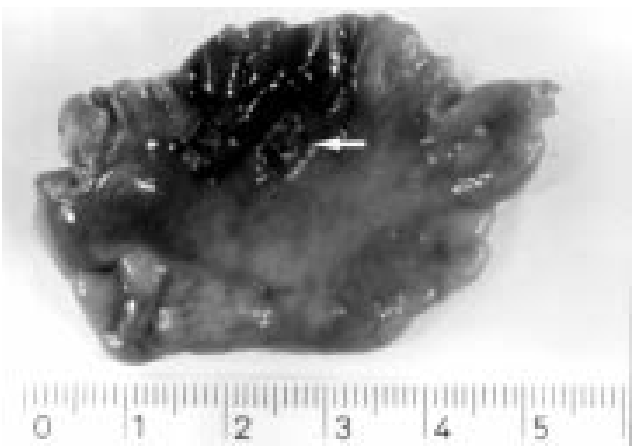

Figure 1 Surgically created bleeding site in the caecum. To induce haemorrhage, the mucosa is scraped (arrow). Subsequently, the bowel is closed with a sealed suture.

\section{Methods}

INTRAVASCULAR CONTRAST AGENT

NC100150 Injection (Nycomed Amersham Imaging, Wayne, Pennsylvania, USA), a colloidal preparation of ultrasmall superparamagnetic iron oxide crystals with an oxidised starch coating, is a new MR blood pool contrast agent with a dose dependent vascular half life ranging between three and four hours; $1 \mathrm{ml}$ of the agent contains $30 \mathrm{mg}$ of iron. It is taken up by macrophages and is metabolised by the liver. The compound has recently undergone successful Phase I human trials which showed it to be effective for the depiction of vascular structures throughout the body. ${ }^{12}$ NC100150 Injection causes considerable shortening of both $\mathrm{T} 1$ and $\mathrm{T} 2$ relaxation times. On heavily $\mathrm{T} 1$ weighted fast $3 \mathrm{D}$ sequences with short echo times (less than $2 \mathrm{~ms}$ ) the $\mathrm{T} 1$ effects predominate.

\section{ANIMAL EXPERIMENTS}

In accordance with state regulations governing animal experiments, six female pigs (mean weight $47 \mathrm{~kg}$ ) were operated on under general

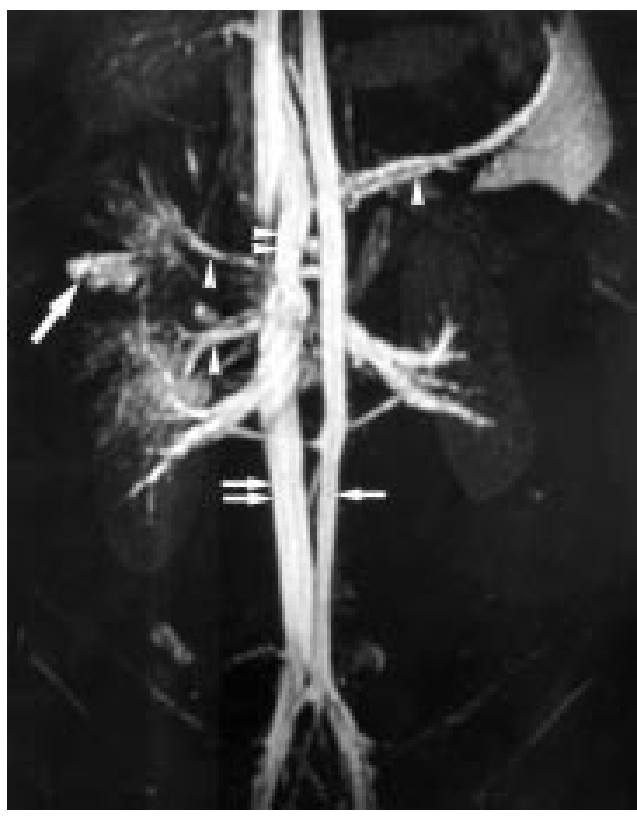

Figure 2 The presence of the intravascular agent causes enhancement of the entire vascular system as shown on this coronal maximum intensity projection image: aorta (arrow), inferior vena cava (double arrow), portal vein (double arrowhead), splenic and mesenteric vessels (arrowheads). A small bleed is visualised in the right upper quadrant, corresponding to the duodenum (large arrow). anaesthesia. Anaesthesia was induced with azaperone $(10 \mathrm{mg} / 10 \mathrm{~kg}$ body weight, Stresnil, Veterinaria AG, Zurich, Switzerland), ketamine $\mathrm{HCl}(150 \mathrm{mg} / 10 \mathrm{~kg}$ body weight, Ketasol, Dr E Gräub, Bern, Switzerland), atropine sulphate $(0.2 \mathrm{ml} / 10 \mathrm{~kg}$ body weight, Atropine $0.1 \%$, Synthetica, Mendrisio, Switzerland), and maintained with halothane $1.5 \%$ (Synmedic AG, Zurich, Switzerland). The animal remained intubated and fully relaxed with pancuronium bromide ( $4 \mathrm{mg} / 2$ hour, Pavulon, Organon Teknika AG, Pfaeffikon, Switzerland) throughout the entire experiment. Between two and four regions in the small bowel and/or colon were tagged and opened. Intraluminal bleeding was surgically induced by scraping the intestinal mucosa (fig 1). To maintain intestinal bleeding, 10000 units of heparin (Liquemin, Roche Pharma, Reinach, Switzerland) were administered intravenously on creation of the intraluminal mucosal lesions. During opening and subsequent closing of the abdomen the surgeon ensured complete haemostasis within the abdominal wall.

Closely monitoring haemodynamic parameters including heart rate, arterial blood pressure, and oxygen saturation, the internally bleeding animal was first studied with scintigraphy and subsequently with the MRI technique. Following completion of the MR protocol, the animals were returned to nuclear medicine for a delayed scan and subsequently sacrificed by injecting $20 \mathrm{ml}$ potassium chloride 15\% (Haenseler AG, Herisau, Switzerland). An autopsy was performed during which the surgically tagged small bowel and colonic segments were harvested and inspected for the presence of haemorrhage.

SCINTIGRAPHY

The in vivo method was used for radiolabelling the pig's blood. ${ }^{13} \mathrm{~A}$ reducing agent, stannous pyrophosphate $(7.5 \mathrm{mg}$, Technescan PYP, Mallinckrodt Medical BV, Petten, The Netherlands) was injected intravenously more than 30 minutes prior to the administration of 640 Mbq of technetium-99m pertechnetate. The animals were placed in the supine position under a gamma camera equipped with a 140 $\mathrm{keV}$ parallel hole collimator (Picker Three Head 3000 Prism, Picker International, Cleveland, Ohio, USA). Three planar images (ventral, left anterior oblique, and right anterior oblique) were acquired at five minute intervals (240 second images) over the first 45 minutes, as well as for the delayed scans following MRI. Single photon emission computed tomography (SPECT) scans (three degrees steps, 40 seconds acquisitions per step) were collected after 45 minutes and at the end of the delayed scan before sacrificing the pigs.

MAGNETIC RESONANCE IMAGING

All MRI was performed on a $1.5 \mathrm{~T}$ MR scanner (Signa EchoSpeed, GE Medical Systems, Milwaukee, Wisconsin, USA). An anteroposterior phased array surface coil was used for signal reception. Based on multiphase gradient recalled echo (GRE) localising images, 3D fast GRE acquisitions were collected using the fol- 
Table 1 Segment by segment analysis of magnetic resonance images compared with autopsy

\begin{tabular}{lllllll}
\hline \multicolumn{7}{c}{ Magnetic resonance imaging } \\
\cline { 2 - 7 } Autopsy & $\begin{array}{l}\text { Definitely } \\
\text { normal }\end{array}$ & $\begin{array}{l}\text { Probably } \\
\text { normal }\end{array}$ & Indeterminate & $\begin{array}{l}\text { Probably } \\
\text { bleeding }\end{array}$ & $\begin{array}{l}\text { Definitely } \\
\text { bleeding }\end{array}$ & Total \\
\hline Normal & 15 & 3 & 0 & 0 & 0 & 18 \\
Bleeding & 0 & 0 & 0 & 4 & 14 & 18 \\
Total & 15 & 3 & 0 & 4 & 14 & 36 \\
\hline
\end{tabular}

lowing parameters: TR $5.2 \mathrm{~ms}$, TE $1.6 \mathrm{~ms}$, flip $30^{\circ}$. One excitation was averaged using a sampling bandwidth of $\pm 62.5 \mathrm{kHz}$. 3D GRE data sets, consisting of 44 contiguous $2.6 \mathrm{~mm}$ sections, were acquired over 30 seconds under apnoea conditions in the coronal plane. Combined with a $32 \mathrm{~cm}$ FOV, a $256 \times 192$ matrix provided an in plane spatial resolution of $1.8 \times 1.2 \mathrm{~mm}$. Following the acquisition of a "native" 3D data set, NC100150 Injection was injected intravenously over 20 seconds in a dose of $5.0 \mathrm{mg} \mathrm{Fe} / \mathrm{kg}$ body weight. The mean injected volume was $7.8 \mathrm{ml}$. Subsequent 3D data sets were collected at five minute intervals for 30 minutes.
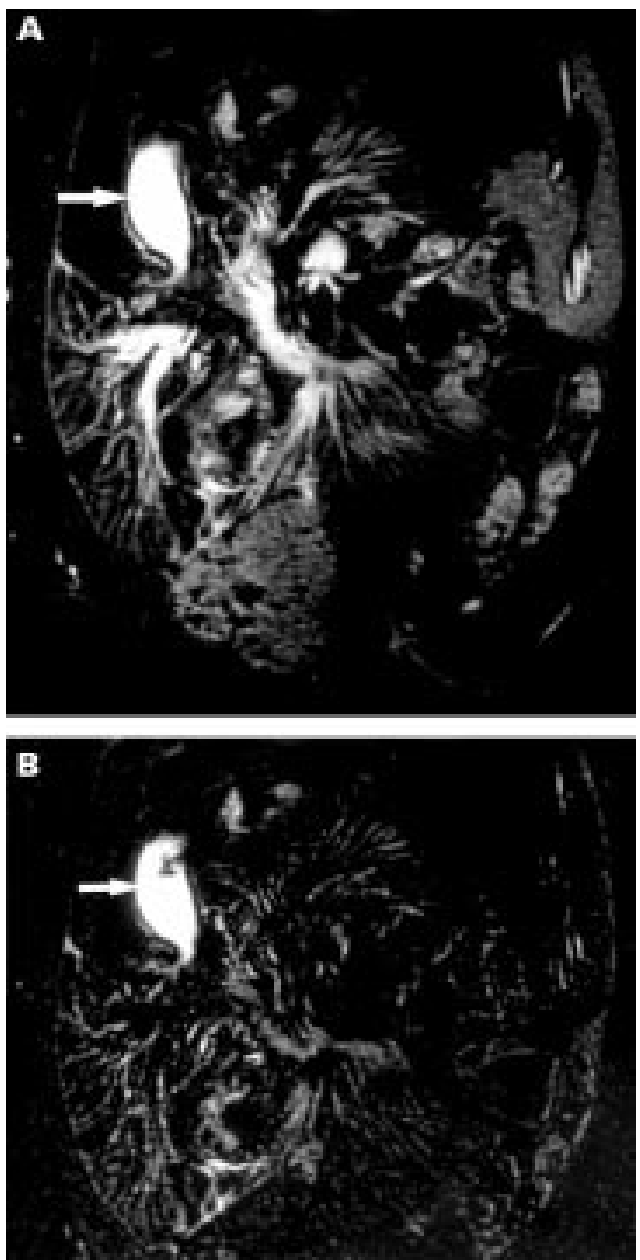

Figure 3 (A) Coronal source image of $3 D$ gradient recalled echo data set collected 10 minutes after administration of the intravascular agent. Blood containing intravascular contrast is already evident in the duodenum (arrow). Note the excellent depiction of the mesenteric vascularity reflecting the presence of the intravascular contrast material. (B) The bleed is seen to even better advantage following subtraction of the first image set, depicting the mesenteric arteries and veins.
IMAGE INTERPRETATION

Analysis of the MRI data was based on individual sections, image subtractions, and maximum intensity projections (MIP). Criteria for identification of a bleeding site included a signal increase exceeding $100 \%$ relative to surrounding structures, and enlarging areas of increased signal over time. Interpretation of the radionuclide scans was based on all available planar and SPECT images, which were reformatted in coronal and axial projections using standard criteria. ${ }^{14-16}$

The studies were interpreted by different readers, each certified and experienced in their respective fields. Both readers knew that the creation of at least one gastrointestinal bleed had been attempted in each animal. The readers were blinded to the exact number of bleeds confirmed at autopsy, the location of the bleeding sites, as well as the reading of the other technique. For analysis, the intestinal tract of the pigs was divided into six regions: stomach/duodenum, proximal small bowel, distal small bowel, caecum/proximal colon, distal colon, and sigmoid colon/rectum. Prior to analysis, both readers were briefed in depth on the intestinal anatomy of pigs. A schematic drawing depicting the location of the different intestinal regions was available to the readers during assessment of all six studies, containing a total of 36 segments. Each segment was rated with each modality on a five point scale: 1 , bleeding definitely not present; 2 , bleeding probably not present; 3, indeterminate; 4, bleeding probably present; 5, bleeding definitely present.

A binomial receiver operator characteristic (ROC) curve was plotted, reflecting the observer's confidence rating. ${ }^{17}$ The area under the corresponding curve was calculated using the trapezoidal rule. ${ }^{18}$ For determination of sensitivity and specificity values, "definite" and "probable" ratings were combined.

\section{Results}

All six animals remained haemodynamically stable throughout the intestinal imaging experiments. Bleeding was confirmed at autopsy in 18/19 surgically created sites. At one site only a minimal amount of blood was identified and was hence considered normal. Thus bleeds were present in 18/36 segments.

Following the intravenous administration of NC100150, the larger abdominal vascular structures were displayed homogeneously bright on 3D data sets (fig 2). Based on the MRI images, "definite" readings regarding the presence of gastrointestinal bleeding were rendered correctly for 29/36 segments. The remaining seven segments received "probable" readings, all of which were correct (table 1). There were no indeterminate readings. The bleeding sites were identified as enlarging areas of increased signal on the coronal 3D data sets (fig 3). Contrast filling of and subsequent movement through the bowel was clearly documented over time (fig 4). Regarding the presence of bleeding, all segments were 
correctly characterised based on the MRI data sets, resulting in $100 \%$ sensitivity and specificity.

Scintigraphy performed less well: only $17 / 36$ segments were correctly characterised as "definitely" bleeding or not bleeding. Two segments were classified as indeterminate, while 10 segments received correct "probable" readings. Interpretation of seven segments (19\%) was false (table 2), resulting in overall sensitivity and specificity values of $78 \%$ and $72 \%$ respectively.
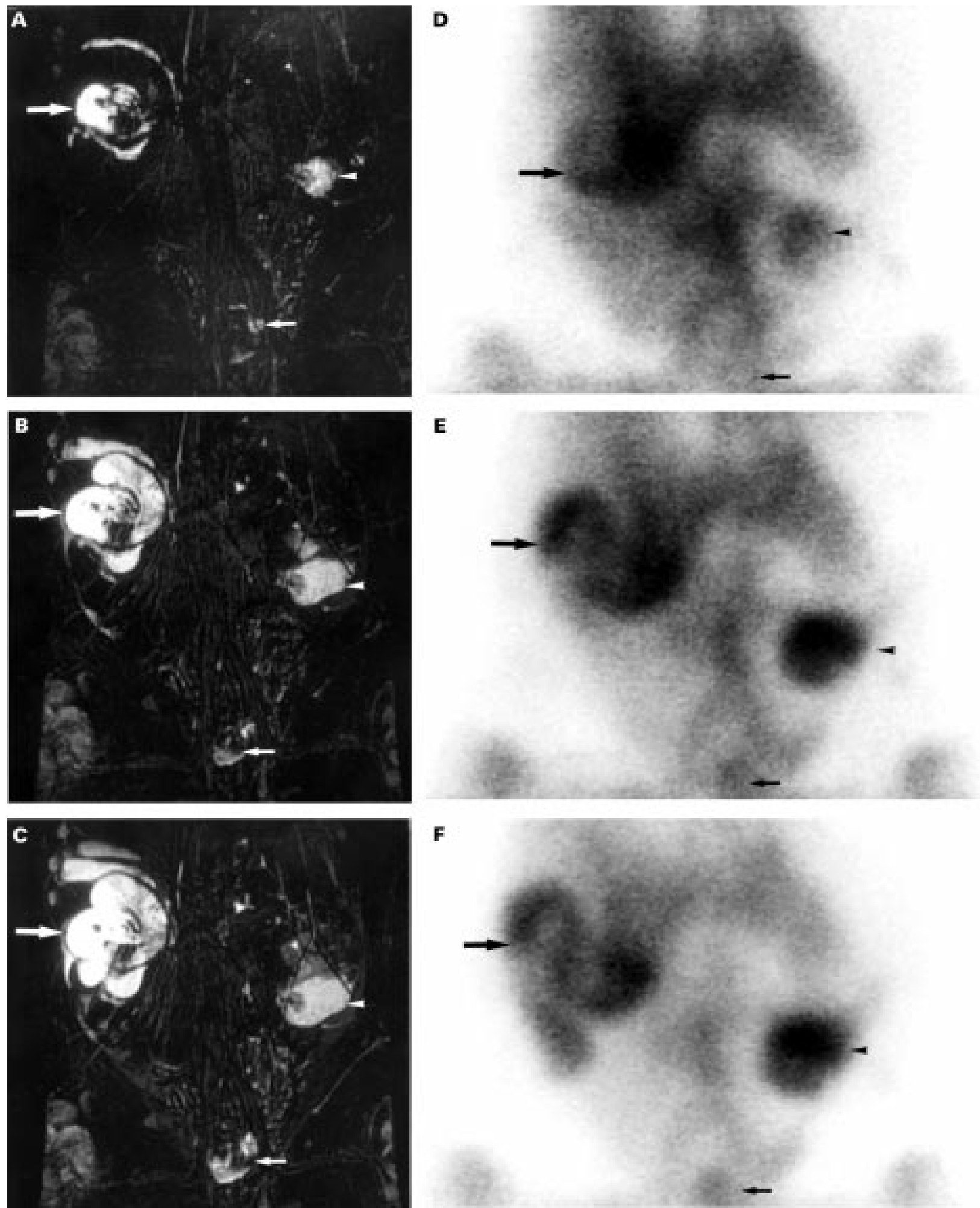

Figure 4 Coronal maximum intensity projection images of $3 D$ gradient recalled echo data sets after subtraction of the first image set collected 10 minutes $(A), 15$ minutes (B), and 20 minutes (C) after administration of the intravascular agent. Increasing contrast accumulation within the small bowel (white large arrow), the caecum (white arrowhead), and the rectum (white small arrow) is evident. Planar scintigraphic images obtained at the same time intervals, 10 minutes (D), 15 minutes (E), and 20 minutes (F) after administration of tagged red blood cells show bleeds in the proximal small bowel (black large arrow) and the caecum (black arrowhead). The rectal bleed (black small arrow) was missed prospectively. 
Table 2 Segment by segment analysis of scintigraphy compared with autopsy

\begin{tabular}{|c|c|c|c|c|c|c|}
\hline \multirow[b]{2}{*}{ Autopsy } & \multicolumn{6}{|c|}{ Scintigraphy } \\
\hline & $\begin{array}{l}\text { Definitely } \\
\text { normal }\end{array}$ & $\begin{array}{l}\text { Probably } \\
\text { normal }\end{array}$ & Indeterminate & $\begin{array}{l}\text { Probably } \\
\text { bleeding }\end{array}$ & $\begin{array}{l}\text { Definitely } \\
\text { bleeding }\end{array}$ & Total \\
\hline Normal & 7 & 6 & 0 & 4 & 1 & 18 \\
\hline Bleeding & 1 & 1 & 2 & 4 & 10 & 18 \\
\hline Total & 8 & 7 & 2 & 8 & 11 & 36 \\
\hline
\end{tabular}

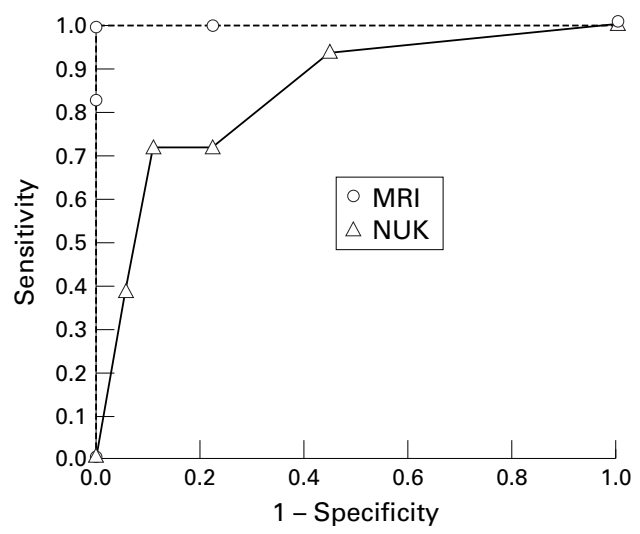

Figure 5 Receiver operator characteristic curves for magnetic resonance imaging (MRI) and scintigraphy (NUK).

Differences in diagnostic performance are summarised in the ROC analysis (fig 5). The excellent performance of 3D MRI is reflected by an area under the curve of close to 0.99 . For scintigraphy the area under the curve was 0.85 .

\section{Discussion}

The proposed MR technique for detection and localisation of gastrointestinal bleeds is based on the recent availability of two components: high performance gradient systems required for fast breathheld 3D MR data acquisitions, and blood pool contrast agents with long intravascular half lives. The agent chosen for this study has been shown to be safe, and is currently undergoing Phase II human trials. Used in combination with fast 3D GRE sequences characterised by short repetition and echo times, the $\mathrm{T} 1$ shortening effect of the contrast agent renders the intravascular signal exquisitely bright, while surrounding tissues maintain a low signal intensity. ${ }^{12}$ The intravascular nature of the agent prevents enhancement outside the vascular system except in those regions with compromised vascular integrity. Thus, areas of active bleeding are readily detected. In addition to reliable detection, the temporal and spatial resolution of the 3D MR data acquisitions proved sufficient to ensure highly accurate localisation of haemorrhage sites within the intestinal tract.

3D MRI in conjunction with an intravascular contrast agent compared favourably with nuclear scintigraphy regarding diagnostic accuracy and the ability to locate the bleeding site in the explored animal model. Reported sensitivity of planar ${ }^{99 \mathrm{~m}} \mathrm{Tc}$ labelled red blood cell (RBC) scintigraphy is $75-94 \%$, with false positive rates as high as $20 \% .{ }^{19}$ Due to the poor spatial resolution inherent to nuclear scintigraphy, ${ }^{10}{ }^{20}$ localisation of the detected bleeding site represents a considerable chal- lenge. Thus accuracy values as low as $52 \%$ have been reported regarding localisation..$^{10}{ }^{20-22}$ In light of these numbers, the segmental analysis of this study revealed excellent results for nuclear scintigraphy. Fourteen of 18 bleeding sites were correctly localised with scintigraphy resulting in sensitivity and specificity values of $78 \%$ and $72 \%$, respectively. Beyond the existence of a reader bias based on the knowledge that at least one bleed was likely to be present in each of the pigs, the relatively good performance of nuclear scintigraphy reflects the use of SPECT in addition to planar imaging. SPECT images are less affected by background activity, and hepatic and splenic tracer accumulation, as well as the uptake of free technetium in the gastric mucosa, all of which can severely restrict the ability to interpret planar scintigraphy. ${ }^{8}$ Furthermore, SPECT allows for multiplanar reformations, thereby enhancing the ability to localise a detected haemorrhage spatially.

3D MRI performed even better, with a $100 \%$ detection and localisation accuracy. All 18 bleeding sites were correctly attributed to their respective intestinal segments. Similarly, all 18 non-bleeding segments were correctly characterised as such. The high anatomic resolution, coupled with the ability to reformat the underlying data interactively in any desired plane, proved adequate for accurate identification and localisation of the affected bowel loops. For 29/36 segments the quality of the data sets was even sufficient to render "definite" readings. Thus the ROC analysis does not only reflect differences in diagnostic accuracy but also in diagnostic confidence between MRI and scintigraphy.

Collected within the confines of a single breathhold lasting 30 seconds, 3D MRI data sets depict the entire abdomen on contiguous 2-3 $\mathrm{mm}$ sections with an in plane resolution of $1.8 \times 1.2 \mathrm{~mm}$. The data sets can be reformatted interactively in any desired plane, permitting a comprehensive analysis of all intestinal structures. The ability to collect $3 \mathrm{D}$ data sets in rapid succession further aided the localisation process. The spread of the contrast containing blood within the intestinal loops is well depicted (fig 6). Shape and course of the loops permits easy differentiation between stomach, small bowel, and colon.

The 3D MRI technique has been shown to be exquisitely sensitive: phantom experiments documented the detection of volumes as little as $0.2 \mathrm{ml} / \mathrm{min}$ of extravasated blood containing the intravascular agent. Compared with scintigraphy, the 3D MRI technique has been shown to be more sensitive with lower flow rates requiring less accumulation of contrast agent. Thus, repetitive 3D MR imaging allowed identification of simulated bleeding with flow rates as small as $0.05 \mathrm{ml} / \mathrm{min} .{ }^{11}$ Similar to scintigraphy, the presence of contrast within the vascular system reduces the conspicuity of small contrast collections in an in vivo environment. To compensate for this effect, subtraction techniques are helpful. Thus, the data set collected immediately following contrast administration can be subtracted from subsequent images. On the 
resultant subtractions only extravasated blood is displayed (fig 3).

Reflecting the vast $\mathrm{T} 1$ shortening effects of the intravascular contrast agent, the abdominal vascularity is clearly seen on $3 \mathrm{D} M R$ images immediately following its intravenous administration. Beyond providing additional spatial information, the simultaneous display of mesenteric vascular morphology may permit identification of individual vessels feeding the intestinal haemorrhage. The surgical model used in this study did not lend itself to assessment of this. Visualisation of the vasculature relative to the bleeding site may also prove useful for choosing and planning the most appropriate therapy. Based on the availability of recently developed techniques for real time catheter and guide wire visualisation, ${ }^{23-25}$ it is conceivable to use the underlying $3 \mathrm{D}$ displays as roadmaps to guide embolisation procedures in patients with intestinal haemorrhage in the future.

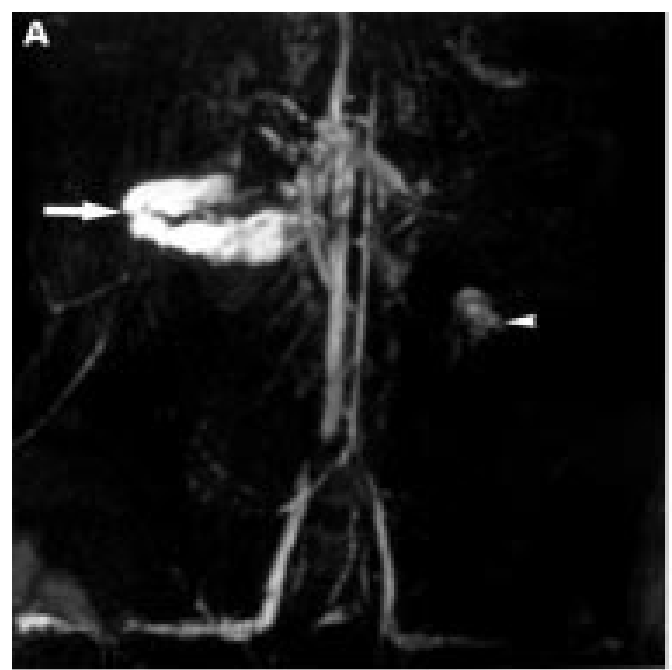

D
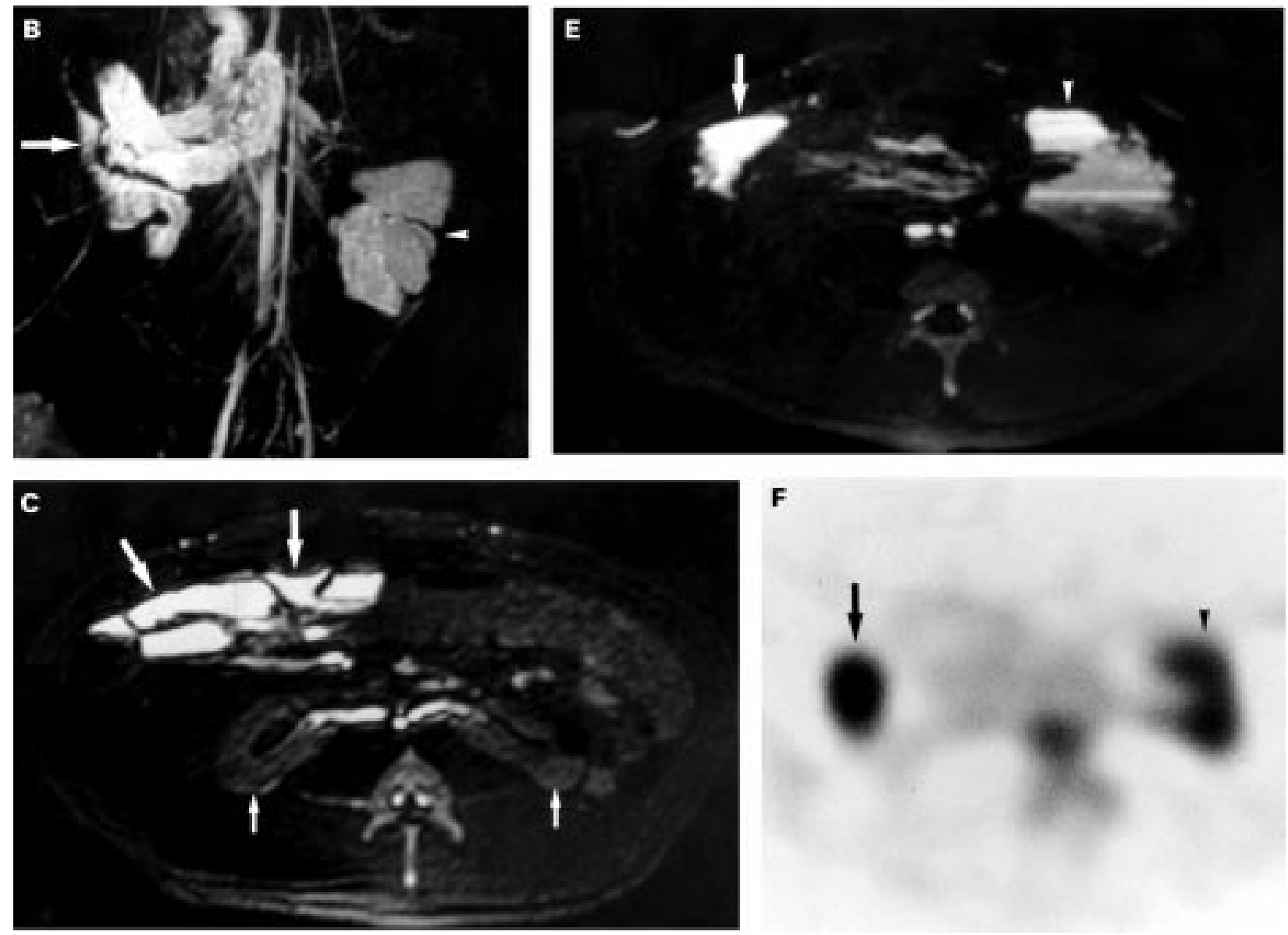

F

Figure 6 Coronal maximum intensity projection images acquired 10 minutes $(A)$ and 20 minutes $(B)$ after administration of the intravascular agent. Increasing contrast accumulation within the proximal small bowel (large arrow) and the caecum (arrowhead) is evident. Corresponding axial magnetic resonance (MR) and single photon emission computed tomography (SPECT) scintigraphic images show the bleeding sites: $(C, D)$ at the level of the kidneys (small arrows), and (E,F) below. Localisation of increased activity on the SPECT images corresponds well with the axial MR images. 
In addition to surpassing the diagnostic performance of scintigraphy, the presented MRI technique seems to overcome limitations inherent to other methods currently used for detecting and localising intestinal haemorrhage. Non-invasiveness, coupled with the long intravascular half life of the blood pool agent, represent distinct advantages over catheter angiography, which is predicated on active bleeding while the iodinated contrast is actually injected. ${ }^{6}$ Thus, repetitive imaging with 3D MRI over several hours enables detection of intermittent haemorrhage, even if it is of venous origin. $3 \mathrm{D} \mathrm{MRI}$ is also considerably less invasive, less costly, and less dependent on operator skill than endoscopy, which is further limited by the inability to assess the entire small bowel. Furthermore, the MRI technique is unaffected by high bleeding rates beyond 1.0 $\mathrm{ml} / \mathrm{min}$, which can complicate endoscopic localisation of the bleeding.

Although the presented study unequivocally shows the potential of the MRI based technique for detecting and localising intestinal haemorrhage, several study limitations need to be considered. Firstly, the surgical technique used did not permit quantification of the induced intestinal bleeds. Expecting the haemorrhage to taper off over the course of the experiment due to hypovolaemia, scintigraphy was performed first. Any advantage related to more severe bleeding in the early stages of the experiment would thus have been reflected in an improved scintigraphic performance. Similarly, the perioperative reduction in bowel peristalsis should have benefited scintigraphy slightly more than MRI. Most limiting from a methodological point of view was the observers' bias introduced by the expectation of at least one bleeding site in each animal. To weaken the effect of this bias, more than one bleeding event was induced in each of the animals.

Clearly the presented MR based approach towards diagnosing gastrointestinal bleeding has limitations. First and foremost, availability of MRI scanners remains limited. The authors are convinced however, that continuous reductions in hardware cost in conjunction with a steady increase in the number of clinically relevant diagnostic indications, including $M R$ angiography or MRCP, will result in a continuous increase in the availability of MRI, even in emergency settings, similar to that seen with CT. A more technical issue relates to the need for breathholding during data collection. For critically ill patients it may be difficult to hold their breath for 30 seconds. For these patients the scan volume could easily be divided into several acquisitions to shorten the breathhold period to less than 10 seconds.

In summary, the presented data illustrate that ultrafast $3 \mathrm{G}$ MR imaging can be used to detect intestinal haemorrhage following intravenous administration of an intravascular contrast agent. Undoubtedly multiple aspects of the proposed technique will require further refinement and testing prior to clinical implementation.

The authors thank Michaela Schmidt for her assistance in The authors thank Michaela Schmidt for her assistance in imaging and preparation of the subtraction images, and Heidi animal anaesthesia.

1 Lieberman D. Gastrointestinal bleeding: initial management. Gastroenterol Clin North Am 1993;22:723-36.

2 Friedman LS, Martin P. The problem of gastrointestinal bleeding. Gastroenterol Clin North Am 1993;22:717-21.

3 Steger AC, Spencer J. Obscure gastrointestinal bleeding. BMF 1988;296:3.

4 Richter JM, Christensen MR, Kaplan LM, et al. Effectiveness of current technology in the diagnosis and management of lower gastrointestinal hemorrhage. Gastrointest Endosc 1995;41:93-8.

5 Whitaker SC, Gregson RH. The role of angiography in the investigation of acute or chronic gastrointestinal haemorrhage. Clin Radiol 1993;47:382-8.

6 Rollins ES, Picus D, Hicks ME, et al. Angiography is useful in detecting the source of chronic gastrointestinal bleeding
of obscure origin. AfR Am $\mathcal{F}$ Roentgenol 1991;156:385-8.

7 Ettorre GC, Francioso G, Garribba AP, et al. Helical CT Ettorre GC, Francioso G, Garribba AP, et al. Helical CT
angiography in gastrointestinal bleeding of obscure origin. AFR Am $\mathcal{F}$ Roentgenol 1997;168:727-31.

8 Wu Y, Seto H, Shimizu M, et al. Sequential subtraction scintigraphy with $99 \mathrm{Tcm}-\mathrm{RBC}$ for the early detection of gastrointestinal bleeding and the calculation of bleeding rates: phantom and animal studies. Nucl Med Commun 1997;18:129-38.

9 Dusold R, Burke K, Carpentier W, et al. The accuracy of technetium-99m-labeled red cell scintigraphy in localizing gastrointestinal bleeding. Am f Gastroenterol 1994;89:3458 .

10 Hunter JM, Pezim ME. Limited value of technetium $99 \mathrm{~m}$-labeled red cell scintigraphy in localization of lower 99m-labeled red cell scintigraphy in localization of 1 .

11 Hilfiker PR, Zimmerman GG, Quick HH, et al. Detection of abdominal and gastrointestinal bleeding with a new MR blood pool agent in conjunction with fast 3D MRI: and experimental study. Proceedings of the International Society for Magnetic Resonance in Medicine, 6. Scientific Meeting and Exhibition, Sydney 1998. New York: International Society for Magnetic Resonance in Medicine (ISMRM), 1998: 306.

12 Wildermuth S, Dubno B, Romanowski BJ, et al. Open-label, phase 1 trial of a new blood pool contrast agent (NC 100150) in 12 healthy volunteers: safety and vascular imaging characteristics. In: Proceedings of the International Society for Magnetic Resonance in Medicine, 6. Scientific MeetSociety for Magnetic Resonance in Medicine (ISMRM), 1998:171.

13 Pavel DG, Zimmer M, Patterson VN. In vivo labeling of red blood cells with $99 \mathrm{mTc}$ : a new approach to blood pool visualization. $\mathcal{F}$ Nucl Med 1977;18:305-8.

14 Smith R, Copely DJ, Bolen FH. 99m Tc RBC scintigraphy: correlation of gastrointestinal bleeding rates with scintigraphic findings. AfR Am f Roentgenol 1987;148:869-74.

15 Winzelberg GG, McKusick KA, Strauss HW, et al. Evaluation of gastrointestinal bleeding by red blood cells labeled in vivo with technetium-99m. F Nucl Med 1979;20:1080-6.

16 Boyd CM, Williamson MR. Technetium-99m RBC bleeding images in normal subjects: confusion with gastrointestinal bleeding sites. Clin Nucl Med 1989;14:202-9.

17 Metz CE. ROC methodology in radiologic imaging. Invest Radiol 1986;21:720-33.

18 Constable RT, Skudlarski P, Gore JC. An ROC approach for evaluating functional brain MR imaging and postprocessing protocols. Magn Reson Med 1995;34:57-64.

19 Suzman MS, Talmor M, Jennis R, et al. Accurate localization and surgical management of active lower gastrointestinal hemorrhage with technetium-labeled gastrointestinal hemorrhage with technetium-labe

20 Voeller GR, Bunch G, Britt LG. Use of technetium-labeled red blood cell scintigraphy in the detection and management of gastrointestinal hemorrhage. Surgery 1991;110: 799-804.

21 Bentley DE, Richardson JD. The role of tagged red blood cell imaging in the localization of gastrointestinal bleeding. Arch Surg 1991;126:821-4.

22 Zuckerman DA, Bocchini TP, Birnbaum EH. Massive hemorrhage in the lower gastrointestinal tract in adults: diagnostic imaging and intervention. Am $\mathcal{f}$ Roentgenol 1993;161:703-11

23 Wildermuth S, Debatin JF, Leung DA, et al. MR imaging-guided intravascular procedures: initial demonimaging-guided intravascular procedures: initial de
stration in a pig model. Radiology 1997;202:578-83.

24 Leung DA, Debatin JF, Wildermuth S, et al. Intravascular MR tracking catheter: preliminary experimental evaluation. AfR Am f Roentgenol 1995;164:1265-70.

25 Ladd ME, Erhart P, Debatin JF, et al. Guidewire antennas for MR fluoroscopy. Magn Reson Med 1997;37:891-7. 